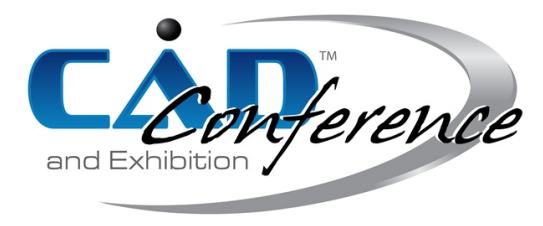

Title:

\title{
Hybrid Line-Arc Toolpath Machining with Corner Transition and Grouping Lookahead Scheme
}

Authors:

Hongyu Ma, hongyuma@amss.ac.cn, University of Chinese Academy of Sciences

Chun-Ming Yuan, cmyuan@mmrc.iss.ac.cn, Academy of Mathematics and Systems Sciences, CAS

Li-Yong Shen, lyshen@ucas.ac.cn, University of Chinese Academy of Sciences

Fengming Lin, linfengming15@mails.ucas.ac.cn, University of Chinese Academy of Sciences

Lixian Zhang, shadowfly12@126.com, Academy of Mathematics and Systems Sciences, CAS

Keywords:

$\overline{\mathrm{CNC}}$, hybrid line-arc toolpath, corner transition, grouping lookahead scheme, feedrate override

DOI: $10.14733 /$ cadconfP.2021.98-102

\section{Introduction:}

As the industry standard for many years, there exists a large amount of numbers of actual workpieces described by the G01 (line segments) and G02/G03 (arcs) codes. In the process of hybrid line-arc toolpath machining, the direction of machining velocity changes suddenly at the junction between two adjacent line-arc toolpaths, which confines the machining efficiency, or causes vibrations harmfully for both the machine and the product quality. To improve the machining efficiency within the precision and acceleration, there are two typical methods: one is to fit the global line segments and arcs into smooth curves and interpolate the fitted curves, another one is to transition the local corners between consecutive segments and interpolate the line-arc paths and the corners.

For the first type method, people often fit the G01 and G02/G03 codes using splines and then interpolate the splines [3]. However, for the spline curves with degree three or higher, the calculations will be complicated for time optimal interpolation. For the second type method, there are further two strategies: one is to design a transition curve to smooth the corners within the tolerance, and then plan the velocities at corners; another one is to merge the above two steps into one step, i.e., design a curve with the time parameter within the machining kinematic constraints and tolerance. For the first strategy, fixed Ferguson splines, arcs, splines or Pythagorean-hodograph corner curves were usually taken as the turning traces [1]. These methods improve the turning velocities in some senses, but the transition paths at the corners are fixed, which limit the turning velocities. The methods for the second strategy improve the machining velocity at corners through one step, and the capability of the machine drives are fully used $[2,5]$. However, the toolpaths in the above methods mainly dealt with the line segments. In practical machining, there are also arc toolpaths, hence, the optimization of the hybrid line-arc toolpath machining need to be considered further. In addition, the lookahead scheme is crucial for improvement of the global machining efficiency, but the computation is time consuming if the number of G01 and G02/G03 codes in lookahead procedure is large. A real-time lookahead algorithm which keeps high machining efficiency is key issue for real-time machining. In practical machining, the feedrate is usually adjusted online by workers for various cutting situations, that is the feedrate override. All the above methods didn't consider 
this situation which is important and indispensable in the CNC system. This motives us to present a transition algorithm which is suitable for hybrid line-arc toolpath.

Corner Transition Algorithm for Hybrid Toolpath:

A key issue in $\mathrm{CNC}$ is to improve the machining feedrate while keeping the machining precision and satisfying the acceleration constraints of the CNC machine. For the consecutive micro-line segments interpolation, the velocities at the junctions of two segments are the bottlenecks for the machining efficiency. Zhang et al. [5] proposed an effective multi-period turning method to improve the feedrate at the junctions using the linear acceleration and deceleration mode. To implement this method, we can approximate the arcs with several even line segments in order to transform the G02/G03 codes to G01 codes (see Fig.1(a)).

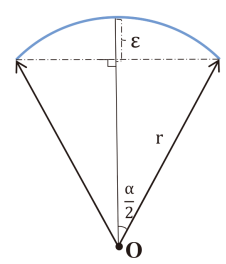

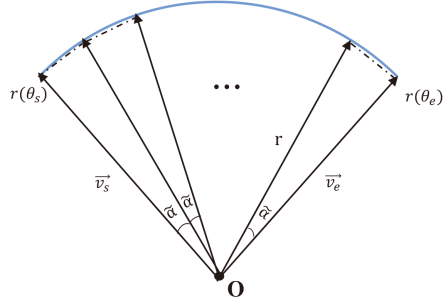

(a)

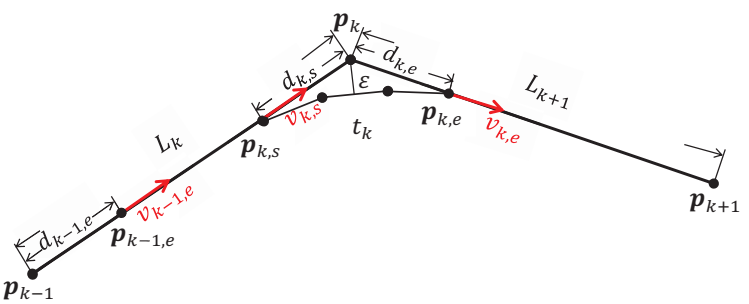

(b)

Fig. 1: (a)Approximate the longest arc with micro-lines; (b)Multi-period turning interpolation at corner.

After arc discretization, we obtain a tool-path with only line segments. We design the corner transition algorithm for line segments. The turning acceleration is chosen based on the principle of bang-bang control (see Fig.1(b)).

In addition to the corner transition interpolation, the interpolation of G01 codes includes the interpolation of line segments. The line segment interpolation algorithm is to calculate the interpolation point sequence on the line segment. We adopt the linear acceleration and deceleration (acc/dec) mode.

Grouping Lookahead Scheme and Feedrate Override Algorithm:

Look-ahead scheme gives an effective way to plan velocity along the whole toolpath. The principle of look-ahead scheme is to ensure that all $\left(v_{k, s}, v_{k, e}\right), k=1, \ldots, n$ are accessible. The accessibility test is in imperative way. During the CNC machining, the interpolation should be able to move in speed $v_{k-1, e}$ to $v_{k, s}$ along the line segment $\mathbf{p}_{k-1, e} \mathbf{p}_{k, s}$; otherwise, $v_{k, s}$ should be modified. Backward accessibility test is similar as the forward accessibility test.

After the accessibility tests, the velocity planning is obtained. However, this lookahead scheme could not be embedded in real machining system directly with the limitation of memory and hardware. There are always up to millions of line segments to be processed in the real world problems. The practical CNC system could store within three thousands line segments, for example, the Blue Sky CNC System.

Assume that the CNC system could store no more than $N_{d}$ line segments. A basic method is to divide the toolpath into groups of $N_{d}$ segments each. For each group including $N_{d}$ line segments, we could build up its look-ahead scheme. However, the machining need to stop and restart at every $N_{d}$ line segments. This way would cut down the machining efficiency and quality. Without losing machining efficiency, we propose the grouping look-ahead scheme to figure out the memory limitation problem.

In order to avoid losing efficiency, the "zero velocity" needs to be eliminated. Given the line length and the acceleration, we could obtain the maximal number $N_{d 0, i}$ of line segments within which the 
maximal velocity may be reached. Then we need to seek the minimum $N_{d 0, i}$ which satisfying the following inequality

$$
2 a_{i, \min } N_{d 0, i} d_{i, \min } \geq v_{\max }^{2}
$$

where $a_{i, \min }=\min \left(A_{x, m}, A_{y, m}, A_{z, m}\right)$.

All the computing works above need to be finished within one machining cycle time. Computing each $N_{d 0, i}$ may waste time. Without losing of generality, we set $d_{i, \min }=\varepsilon$ which can be read from the $\mathrm{G}$ codes. Then a fixing number for $N_{d 0, i}$ is obtained as

$$
N_{d 0}=\left\lceil v_{\max }^{2} /\left(2 a_{i, \min } \varepsilon\right)\right\rceil .
$$

In the segments $i N_{d}-i N_{d 0}, \ldots, i N_{d}-(i-1) N_{d 0}$, the velocity is reachable at each point (See Fig. 2). Therefore, we maintain the larger one among the two velocity values of two groups. Let $v_{k, s}^{*}$ record the ex-turning velocity obtained by the last look-ahead computing, similarly as $v_{k, e}^{*}, t_{k}^{*}$; $v_{k, s}, v_{k, e}$ and $t_{k}$ are the latest value.

$$
\begin{gathered}
v_{k, s}=\max \left\{v_{k, s}^{*}, v_{k, s}\right\}, v_{k, e}=\lambda_{k} v_{k, s}, t_{k}=\eta_{k} v_{k, s} \\
k=i N_{d}-i N_{d 0}, \ldots, i N_{d}-(i-1) N_{d 0} .
\end{gathered}
$$

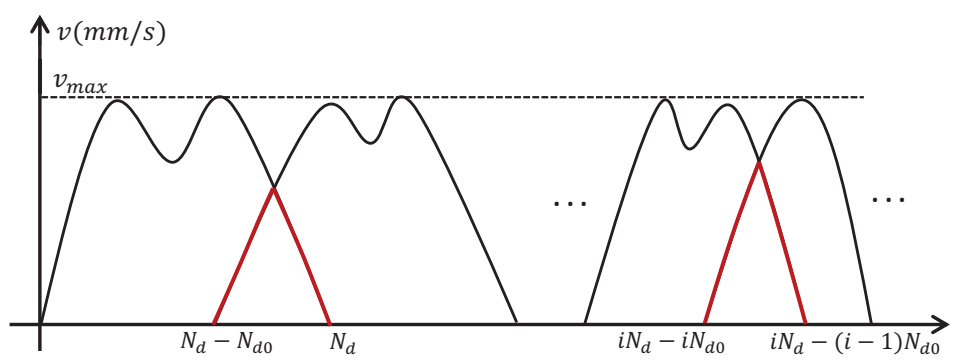

Fig. 2: Grouping method.

By the linear acc/dec mode, one can show that the interpolation value $\left(v_{k, s}, v_{k, e}, t_{k}\right)$ is the same as the value obtained by the grouping method. Therefore, the grouping method gives a feasible way to be implemented into the practical applications without losing any efficiency.

In CNC machining, the feedrate override occurs frequently. For example, the machining process needs to stop when workers find something abnormal. The velocity should decrease from current speed to zero as soon as possible within the machining ability. Or if workers wish to slow down the machining when the vibration happens, that means the velocity should be reduced to the desired velocity quickly. In other cases, the velocity is better to increase quickly when the maximal velocity increase. Therefore, if the machine parameters change, the above look-ahead scheme is not suitable for the new system. However, we expect that the system could response the change as early as possible. The quick respond method for feedrate override is important for $\mathrm{CNC}$ machining.

We give a method to response the feedrate override quickly. Our method could deal with kinds of cases including system pause, feedrate increase and decrease. In order to avoid acceleration override, it is not responded at every corner. The feedrate override is handled on the line segments. The interpolation is under acc/dec mode.

Let $\mathbf{p}_{k, a}$ denote the interpolating point on the line $\mathbf{p}_{k-1} \mathbf{p}_{k}$ when the feedrate $v_{\text {max }}$ is adjusted. At point $\mathbf{p}_{k, a}$, the current velocity $v_{k, a}$ and the interpolation distance $d_{k, a}$ from $\mathbf{p}_{k-1}$ are recorded during the interpolation (See Fig.3).

Experiments and Results: 


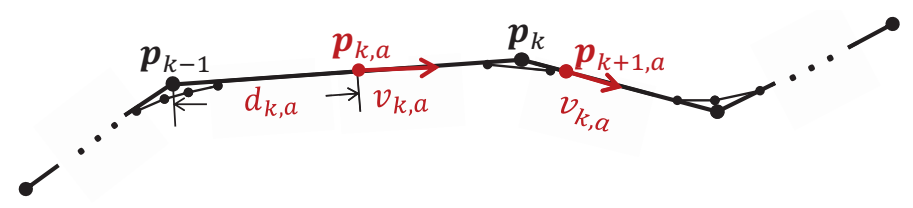

Fig. 3: Feedrate override.

Our method is embed into Blue Sky NC System of Shenyang Institute of Computing Technology Co. Ltd, CAS. We make simulation experiments for several data provided by Guangxi Yuchai Machinery Group Co., Ltd and compare the interpolation time between different algorithms: Yuchai's method provided by Guangxi Yuchai Machinery Group Co., Ltd, Zhang XH's method in [4] and our method (hereinafter referred to as $Y C$ method and $Z X H$ method).

The machining parameters are: the maximal acceleration of each axis is $1600 \mathrm{~mm} / \mathrm{s}$, the maximal velocity (feedrate) is $250 \mathrm{~mm} / \mathrm{s}$, the machining cycle time is $2 \mathrm{~ms}$, and the machining error is $0.1 \mathrm{~mm}$. The $N_{d 0}$ here can be set as 30 .

The machining test of our method is implemented on six models of machining products (two of them is shown in Fig.4. A real machining situation is shown in Fig 5.

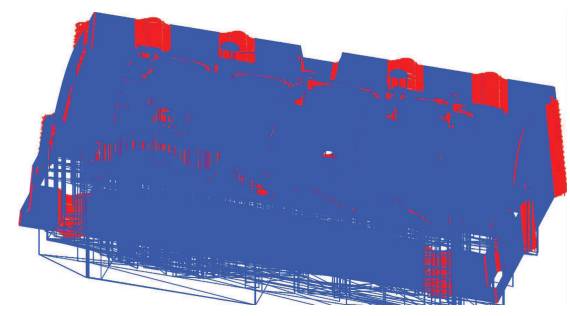

The b684 Model.

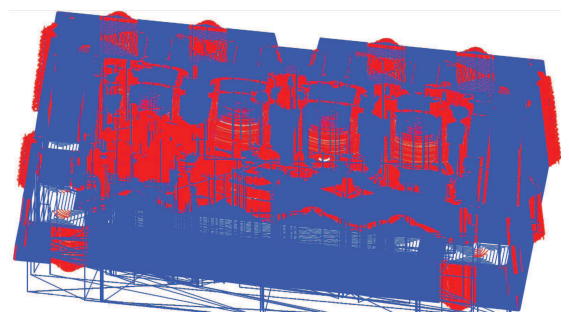

The XM-E8 Model.

Fig. 4: Machining Models: Blue lines represent the G01 codes, and red curves represent G02/G03 arcs.

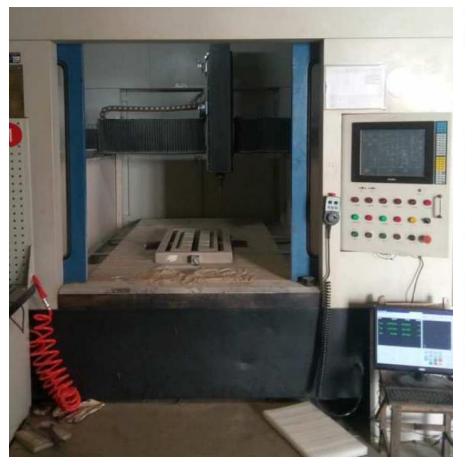

(a) The machine

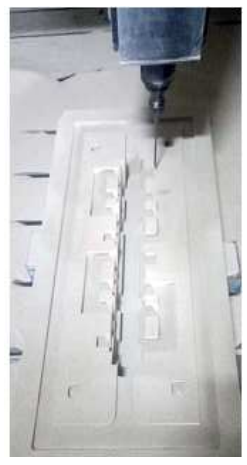

(b) The workpiece.

Fig. 5: The experimental CNC machine and the workpiece.

The comparison of whole simulated machining time is shown in Table 1. The data volume is evaluated by the numbers of line segments. With the same machining parameters, compared with real YC's method, 
our method could cut down the overall interpolation time by $150 \% \sim 200 \%$, while the ZXH's method by $30 \% \sim 50 \%$.

Table 1: Interpolation time comparison between different interpolation algorithms.

\begin{tabular}{|c|c|c|c|c|c|c|c|}
\hline \multirow{2}{*}{ Models } & toolpath & Data & \multicolumn{3}{|c|}{ Overall time (min) } & \multicolumn{2}{c|}{ Velocity improvement (\%) } \\
\cline { 4 - 8 } & length(m) & Volume & YC & ZXH & our & our vs. YC & ZXH vs. YC \\
\hline b6r3 & 161.361 & 6856 & 40 & 27.40 & 13.84 & 189.02 & 45.99 \\
\hline XM-B6R3 & 160.548 & 6115 & 45 & 30.35 & 15.17 & 196.64 & 48.27 \\
\hline b684 & 2189.99 & 953892 & 1627 & 1154.41 & 600.91 & 170.76 & 40.94 \\
\hline XM-E8 & 1526.88 & 274155 & 639 & 447.96 & 233.73 & 173.39 & 42.65 \\
\hline e16 & 2037.45 & 150412 & 590 & 445.15 & 224.42 & 162.90 & 32.54 \\
\hline XM-E16 & 2038.54 & 150127 & 584 & 442.59 & 227.15 & 156.99 & 31.95 \\
\hline
\end{tabular}

\section{Conclusion:}

For the hybrid line-arc toolpath interpolation, the turning velocity improvement at the corners make sense for this goal. We proposed the corner transition interpolation method for the hybrid line-arc toolpath machining with grouping look-ahead scheme, which satisfies the machining error allowance, realtime performance, acceleration and feedrate constraints. In certain senses, the turning velocity is optimal. The grouping look-ahead scheme predetermines the global accessibility efficiently. As a result, the vibration caused by the sudden velocity change is weakened. The implemented CNC machining results of this algorithm show the advantages of the method.

Acknowledgment:

This work is partially supported by Beijing Natural Science Foundation (No. Z190004), National Natural Science Foundation of China under Grants 11688101 and National Center for Mathematics and Interdisciplinary Sciences. We thank Shenyang Institute of Computing Technology Co. Ltd, CAS for the simulation in their Blue Sky NC System and Guangxi Yuchai Machinery Group Co., Ltd for their experimental model data.

\section{References:}

[1] Farouki, R.T.; Pelosi, F.; Lucia Sampoli, M.: Optimization of corner blending curves. ComputerAided Design, 117, 102739, 2019. http://doi.org/10.1016/j.cad.2019.102739.

[2] Tajima, S.; Sencer, B.: Global tool-path smoothing for cnc machine tools with uninterrupted acceleration. International Journal of Machine Tools \& Manufacture, 121, 81-95, 2017. http: //doi.org/10.1016/j.ijmachtools.2017.03.002.

[3] Yuen, A.; Zhang, K.; Altintas, Y.: Smooth trajectory generation for five-axis machine tools. International Journal of Machine Tools \& Manufacture, 71, 11-19, 2013. http://doi.org/10.1016/j . ijmachtools.2013.04.002.

[4] Zang, X.; Yu, D.; Yang, D.; Hu, Y.; Han, W.: Corner curve transition interpolation algorithm for high speed machining of micro-line segment. Chinese Journal of Mechanical Engineering, 46(19), 183-191, 2010. http://doi.org/10.3901/JME.2010.19.183.

[5] Zhang, L.; Sun, R.; Gao, X.S.; Li, H.: High speed interpolation for micro-line trajectory and adaptive real-time look-ahead scheme in cnc machining. Science China Technological Sciences, 54(6), 14811495, 2011. http://doi.org/10.1007/s11431-011-4329-9. 